\title{
Evaluación y análisis sensorial en la reducción de porcentaje de azúcar en leches saborizadas de consumo popular, mediante la reformulación de las mismas
}

\author{
José Miguel Bonilla Portillo ${ }^{1}$ - Franklin Kennedy Ventura Villegas ${ }^{2}$ - Felipe Javier Alvarado Martínez $z^{3}$
}

Recepción: 12/02/2019 Aceptación: 26/05/2019

\section{Resumen}

La leche saborizada es uno de los productos lácteos más consumidos en El Salvador; sin embargo, por el alto contenido de azúcar, es un alimento perjudicial para el consumidor. Ante esto, se desarrolló una alternativa de formulación y producción de leche saborizada con bajo contenido de azúcar. Se trabajaron tres fórmulas de leche saborizada (chocolate, fresa y caramelo) usando diferentes porcentajes de agua, suero dulce, azúcar, estabilizante y saborizante.

Se encontró que las leches que tenían un mayor porcentaje de suero dulce requerían menos cantidad de azúcar en comparación a aquellas que en su formulación llevan menos porcentaje de suero dulce. Las variables que se midieron fueron porcentaje de azúcar, porcentaje de suero dulce y aceptación del producto.

La alternativa de un producto con bajo porcentaje de azúcar genera un incremento en la productividad del sector lechero, ofreciendo al consumidor un valor agregado, al ser considerarlo un producto saludable en comparación a la leche saborizada tradicional; además de postularse como una oportunidad de nuevos negocios y mercados. El objetivo principal de la investigación fue brindar alternativas en la reducción de azúcar en leche saborizada de consumo popular. Para ello se desarrollaron diferentes procesos de leche saborizada, utilizando porcentajes de edulcorantes diferentes para estandarizar fórmulas con leches saborizadas que mantuvieran el sabor característico y con menos porcentajes de azúcar.

Los resultados obtenidos muestran la aceptación por parte de los consumidores teniendo estas una reducción del 15\% de contenido calórico en los productos propuestos, en comparación con las leches de mayor consumo; estos resultados son de beneficio para la salud de los consumidores.

Palabras clave: Estabilizador, pasteurización, ingesta, edulcorantes, saborizantes, suero dulce.

\begin{abstract}
Flavored milk is one of most consumed dairy products in El Salvador; however, due to its high sugar content, it is a harmful food for the consumer. In view of this, an alternative and production of flavored milk with low sugar content was developed. Three flavored milk formulas (chocolate, strawberry, and caramel) were developed using different percentages of water, sweet whey, sugar stabilizer, and flavoring.

It was found that milks with a higher percentage of sweet whey required less sugar compared to those with a lower percentage of sweet whey. The variables measured were sugar percentage, sweet whey percentage and product acceptance.

The alternative of product with a low percentage of sugar generates an increase in the productivity of the dairy sector, offering the consume an added value, as it is considered a healthy product compared to the traditional flavored milk, in addition to being postulated as an opportunity for new business and markets. The main objective of the research was to provide alternatives in the reduction of sugar I flavored milk for popular consumption. Different flavored milk processes were developed, using different percentages of sweeteners to standardize formulas with flavored milks that maintained the characteristic flavor and with lower percentages of sugar.
\end{abstract}

Then obtained results show the acceptance on the part of the consumer having these a reduction of $15 \%$ of caloric content in the proposed products, in comparison with the milks of a greater consumption; these results are of benefit for the health of the consumers.

Key words: Stabilizer, pasteurization, ingestion, sweeteners, flavors, sweet whey.

1. Ingeniería agronómica y Maestría en Asesoría Educativa, Docente tiempo completo, Universidad Católica de El Salvador, El Salvador; email: jose.bonilla@catolica.edu.sv

2. Técnico en Procesamiento de Alimentos, Docente tiempo completo, Universidad Católica de El Salvador, El Salvador; email: franklin.ventura@catolica.edu.sv 3. Técnico en Procesamiento de Alimentos, Docente tiempo completo, Universidad Católica de El Salvador, El Salvador; email: felipe.alvarado@catolica.edu.sv 


\section{Introducción}

Los habitantes de los países desarrollados y subdesarrollados consumimos ahora más calorías que antaño. La obesidad, definida como un exceso de grasa corporal, es el resultado de un balance de energía, y es la forma más frecuente de malnutrición.

El consumo de calorías ha aumentado un promedio de 150 a 300 calorías por día. Casi un $50 \%$ de este incremento proviene de calorías líquidas, en particular, de bebidas azucaradas, elaboradas principalmente con fructosa. Se considera que el consumo de esta contribuye a la mayor tasa de obesidad de los países industrializados, al existir una relación temporal, paralela y directa entre su consumo y el incremento de la obesidad. En algunos grupos de población de Estados Unidos, la ingesta de estas bebidas supone el 15\% de las calorías diarias recomendadas.

Por otro lado, el azúcar es un ingrediente esencial de nuestro día a día. Supone un rápido e importante aporte energético. Forma parte de la hidratación oral, tan relevante en los deportistas. Puede ayudar a las personas mayores y a enfermos con falta de apetito a consumir alimentos nutricionalmente muy deseables.

Sin embargo, parece necesario reflexionar sobre las proporciones epidémicas que está alcanzando la obesidad, con el objetivo de establecer las estrategias terapéuticas más oportunas; y en este contexto, analizar el papel de ciertos alimentos, como es el caso de las bebi- das azucaradas. También, su evidencia disponible para, a partir de ello, ofrecer mensajes poblacionales de moderación/restricción de su consumo. Por tanto, la ingesta de azúcar, por encima de las cantidades recomendadas, se asocia a un mayor riesgo de padecer enfermedad cardiovascular.

La preocupación por la alimentación y la nutrición es mayor, esto debido al incremento de personas con diagnóstico de obesidad. En países desarrollados ha alcanzado dimensiones epidémicas y continuas en aumento. Existen múltiples factores que influyen en la incidencia de obesidad, y junto con el descenso de actividad física, el exceso de ingesta de alimentos inadecuados juega un papel preponderante en la aparición de este problema de salud pública.

El aumento en la oferta y demanda de bebidas azucaradas es cada día mayor; sin embargo, estas bebidas por su alto contenido de azúcares resultan ser un verdadero problema a futuro para las personas que las ingieren. Los porcentajes de azúcares que una persona necesita en el día, acorde a la Organización Mundial de la Salud (OMS), no deben superar el 10\% de la ingesta calórica total diaria, lo que equivale a $50 \mathrm{~g}$ de azúcar.

Por tal razón fue necesario evaluar y analizar de forma sensorial la reducción del porcentaje de azúcar en leches saborizadas de consumo popular. 


\section{Materiales y Métodos}

La investigación que se realizó fue de manera experimental, ejecutando cada uno de los procesos en planta piloto de lácteos de la $\mathrm{Fa}$ cultad Multidisciplinaria de Ilobasco, departamento de Cabañas, Universidad Católica de El Salvador, El Salvador.

Primero, se determinaron los tres tipos de leches saborizada de mayor venta comercial en el país. Una vez seleccionada una de esas variedades, se evaluaron sus componentes nutricionales.

Luego se desarrollaron fórmulas alternativas, disminuyendo el porcentaje de azúcar de la variedad saborizada seleccionada. Dentro de la planta de lácteos se trabajaron prototipos de leche con tres sabores. Seguidamente, se realizaron pruebas sensoriales a los productos elaborados, con el fin de determinar la aceptabilidad de los consumidores. Al finalizar las pruebas sensoriales, se analizaron los resultados pertinentes. Cabe añadir que se reformularon los productos, tomando como base los resultados obtenidos al momento de realizar las pruebas sensoriales. Posteriormente, se desarrollaron nuevas y diversas pruebas sensoriales con su respectivo análisis de resultados hasta obtener características aceptables por los consumidores.

\section{a. Proceso de elaboración de leche saborizada}

- Recepción de materia prima

- Análisis de rápidos

- Pesaje de ingredientes

- Descremado de la leche

- Colocar la leche en una olla y elevar la temperatura.

- Mezclar: azúcar, estabilizador, leche en pol- vo y colorante. Agregar a $50^{\circ} \mathrm{C}$ a la leche.

- Pasteurización a $65^{\circ} \mathrm{C}$ por 20 minutos.

- Enfriar la mezcla a $20^{\circ} \mathrm{C}$.

- Almacenamiento a $4^{\circ} \mathrm{C}$ por 13 horas.

- Agregar el sabor y mezcle durante 5 minutos.

- Envase y almacenado

- Distribución

b. Formulación de leche saborizada reducida en azúcar

- Reducción de porcentaje de leche en polvo.

- Se cambió la leche en polvo entera por leche en polvo descremada.

- Se aumentó el porcentaje de estabilizador.

- Se agregó suero dulce.

- Se agregó agua.

c. Flujograma para leche saborizada (ver figura 1).

\section{Resultados y Discusión}

Para el estudio se abordó a una muestra de 70 estudiantes, pertenecientes a la Escuela de Alimentos de la Facultad Multidisciplinaria de Ilobasco de la Universidad Católica de El Salvador (ver figura 2).

Se evidencia que la lecha marca Salud tiene mayor porcentaje de consumo (64\%); en segundo lugar, se encuentra la marca Shakalaka con un $21 \%$, siendo la menos consumida la marca Sula (2\%).

En cuanto a sabor preferido por los consumidores, un $48 \%$ de ellos aseguró que consume leche con sabor a chocolate, seguido de leche con sabor a fresa (38\%). Los sabores menos consumidos fueron el banano (10\%) y caramelo (4\%) (ver tabla 1 ). 


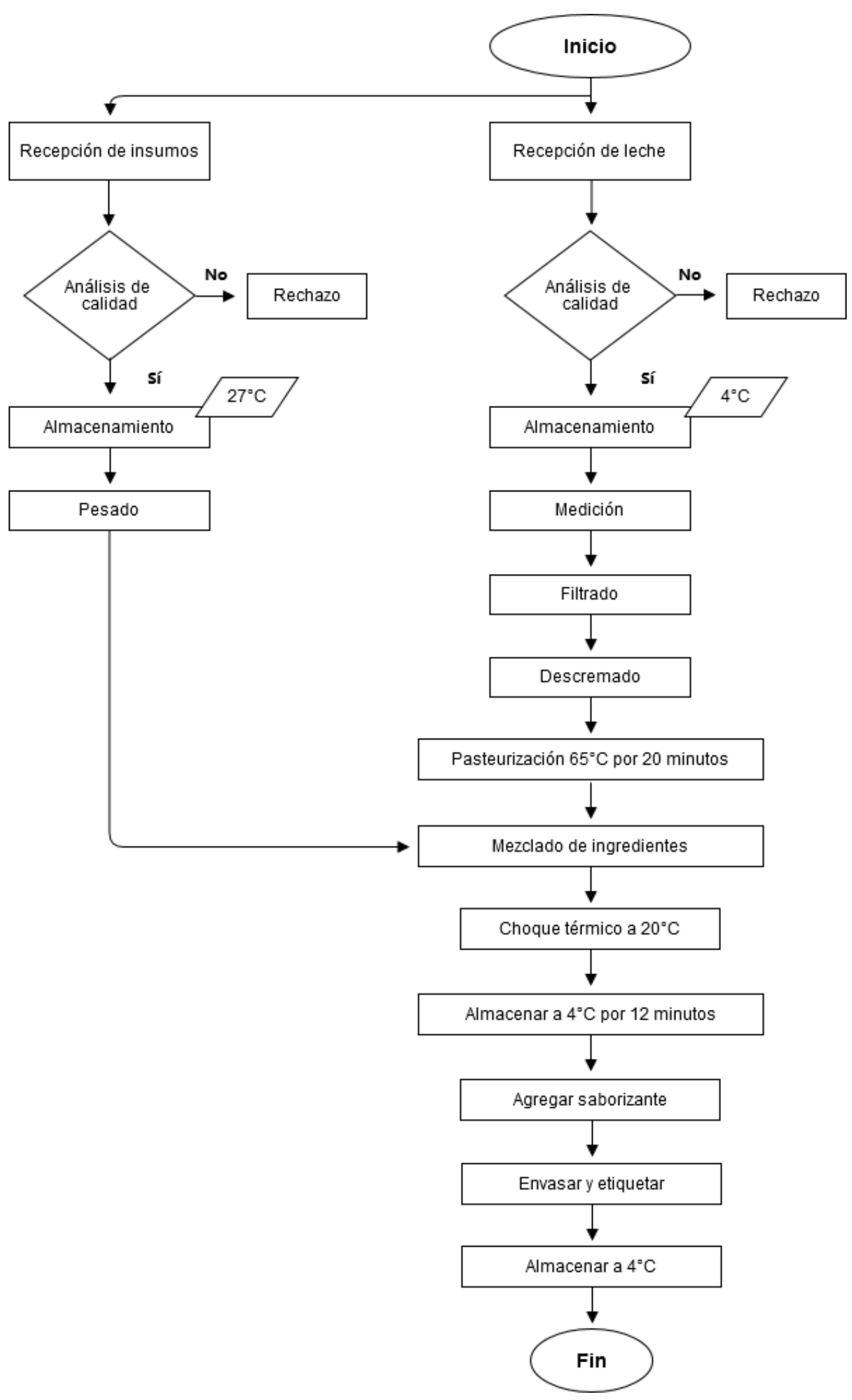

Figura 1. Flujograma para la leche saborizada.

Evaluación y análisis sensorial en la reducción de porcentaje de azúcar en leches saborizadas de consumo popular, mediante la reformulación de las mismas

José M. Bonilla - Franklin K. Ventura - Felipe J. Alvarado. El Salvador 


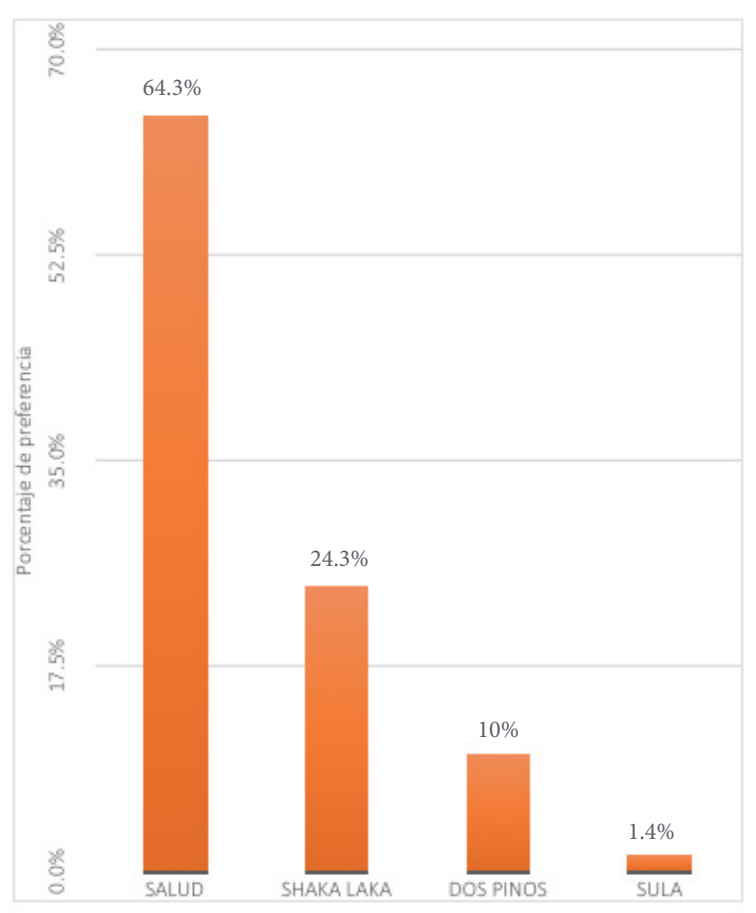

Respecto a los atributos sensoriales (color y sabor) de la leche sabor a chocolate, más del $50 \%$ de las personas consultadas manifestaron que les agrada muchísimo. El factor consistencia posee un bajo nivel de aceptación (30\%), mientras que el olor es aceptable para un $60 \%$ (ver figura 3 ).

Los atributos sensoriales y la consistencia de la leche sabor a fresa son agradables para más de la mitad de las personas consultadas. Al $17 \%$ de ellas les desagrada el olor y el color de la leche saborizada; mientras que a un $9 \%$ le desagrada la consistencia (ver figura 4).

Figura 2. Marcas de leche saborizada de ma-

yor consumo en el mercado nacional.

Tabla 1. Datos nutricionales de leches saborizadas comerciales (Porción de 240g)

\begin{tabular}{|c|c|c|c|c|}
\hline \multirow{2}{*}{ Nutrientes } & \multicolumn{4}{|c|}{ Marcas de leche estudiadas } \\
\cline { 2 - 5 } & Salud & Shaka laka & Dos pinos & Sula \\
\hline Calorías & $130 \mathrm{kcal}$ & $178 \mathrm{kcal}$ & $193 \mathrm{kcal}$ & $190 \mathrm{kcal}$ \\
\hline Grasa total & $3 \mathrm{~g}(5 \%)$ & $5 \mathrm{~g}$ & $5 \mathrm{~g}(8 \%)$ & $4.5 \mathrm{~g}(7 \%)$ \\
\hline Grasa saturada & $1.5 \mathrm{~g}(8 \%)$ & $3.2 \mathrm{~g}$ & $1.3 \mathrm{~g}(7 \%)$ & $3 \mathrm{~g}(15 \%)$ \\
\hline Colesterol & $5 \mathrm{mg}(4 \%)$ & $12 \mathrm{mg}$ & $\mathrm{NA}$ & $10 \mathrm{mg}(3 \%)$ \\
\hline Sodio & $180 \mathrm{mg}(7 \%)$ & $120 \mathrm{mg}$ & $98 \mathrm{mg}(4 \%)$ & $160 \mathrm{mg}(7 \%)$ \\
\hline Carbohidratos & $21 \mathrm{~g}(7 \%)$ & $29 \mathrm{~g}(7 \%)$ & $30 \mathrm{~g}$ & $32 \mathrm{~g}(11 \%)$ \\
\hline Fibra dietética & $0 \%$ & $0 \%$ & $0 \%$ & $0 \%$ \\
\hline Azúcar & $\mathrm{ND}$ & $\mathrm{ND}$ & $\mathrm{ND}$ & $30 \mathrm{~g}$ \\
\hline Proteína & $4 \mathrm{~g}$ & $5 \mathrm{~g}$ & $7 \mathrm{~g}(14 \%)$ & $6 \mathrm{~g}(12 \%)$ \\
\hline Vitamina A & $130 \mathrm{IU}(6 \%)$ & $19 \%$ & $150 \mu \mathrm{g}(19 \%)$ & $(20 \%)$ \\
\hline Vitamina C & $1 \mathrm{mg}(1 \%)$ & $14 \%$ & $10 \mathrm{mg}(17 \%)$ & $(45 \%)$ \\
\hline Calcio & $100 \mathrm{mg}(10 \%)$ & $12 \%$ & $270 \mathrm{mg}(34 \%)$ & $(25 \%)$ \\
\hline Hierro & $1 \mathrm{mg}(2 \%)$ & $14 \%$ & $1.2 \mathrm{mg}(9 \%)$ & $(10 \%)$ \\
\hline
\end{tabular}

Fuente: Tabla de Composición de Alimentos para Centroamérica del INCAP (2012). 

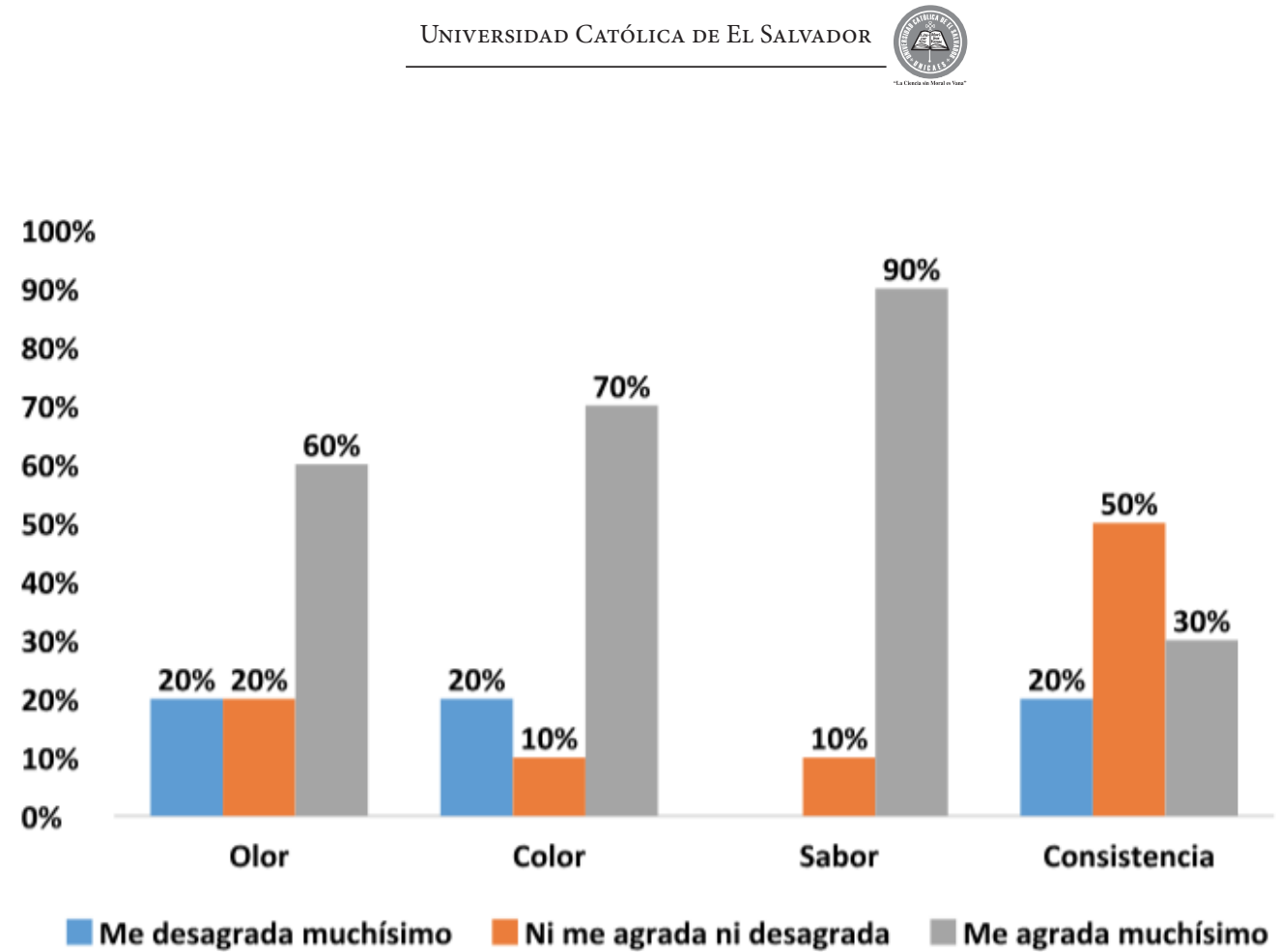

Figura 3. Evaluación sensorial de prototipo sabor chocolate.

\section{$100 \%$}

$90 \%$

$80 \%$

$70 \%$

$60 \%$

$50 \%$

$40 \%$

$30 \%$

$20 \%$

$10 \%$

$0 \%$
$59 \%$

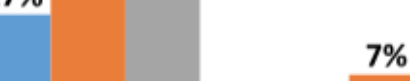

$7 \%$

$93 \%$
$59 \%$

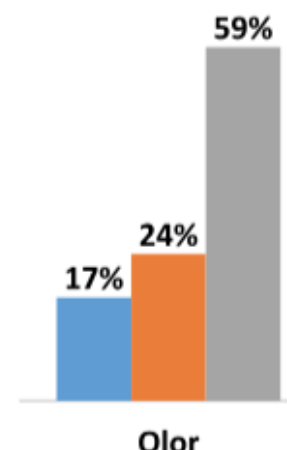

Olor
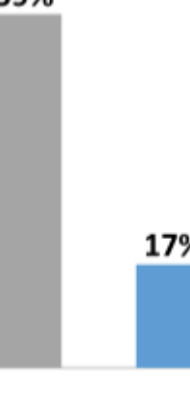

$26 \%$ $17 \%$

Color

Me desagrada muchísimo

Ni me agrada ni desagrada

Me agrada muchísimo

Figura 4. Evaluación sensorial de prototipo sabor fresa. 
Una vez realizadas las pruebas sensoriales finales de la leche saborizada, se obtuvieron los datos que se pueden observar en la figura 5 .

Para la leche sabor a chocolate, un $71 \%$ de a los consumidores acepta su sabor; mientras que un $86 \%$ aprueba su color y su olor. Para el factor consistencia, se obtiene un $100 \%$ de aceptación, dando como conclusión que la leche sabor a chocolate es aceptada.
La leche sabor a fresa es aceptada por los consumidores en un porcentaje arriba de $75 \%$ respecto a los elementos de olor, color, sabor y consistencia. A ningún consumidor que participó del estudio le desagradó $(0 \%)$. En conclusión, la leche sabor a fresa tiene la aprobación de los consumidores (ver figura 6).

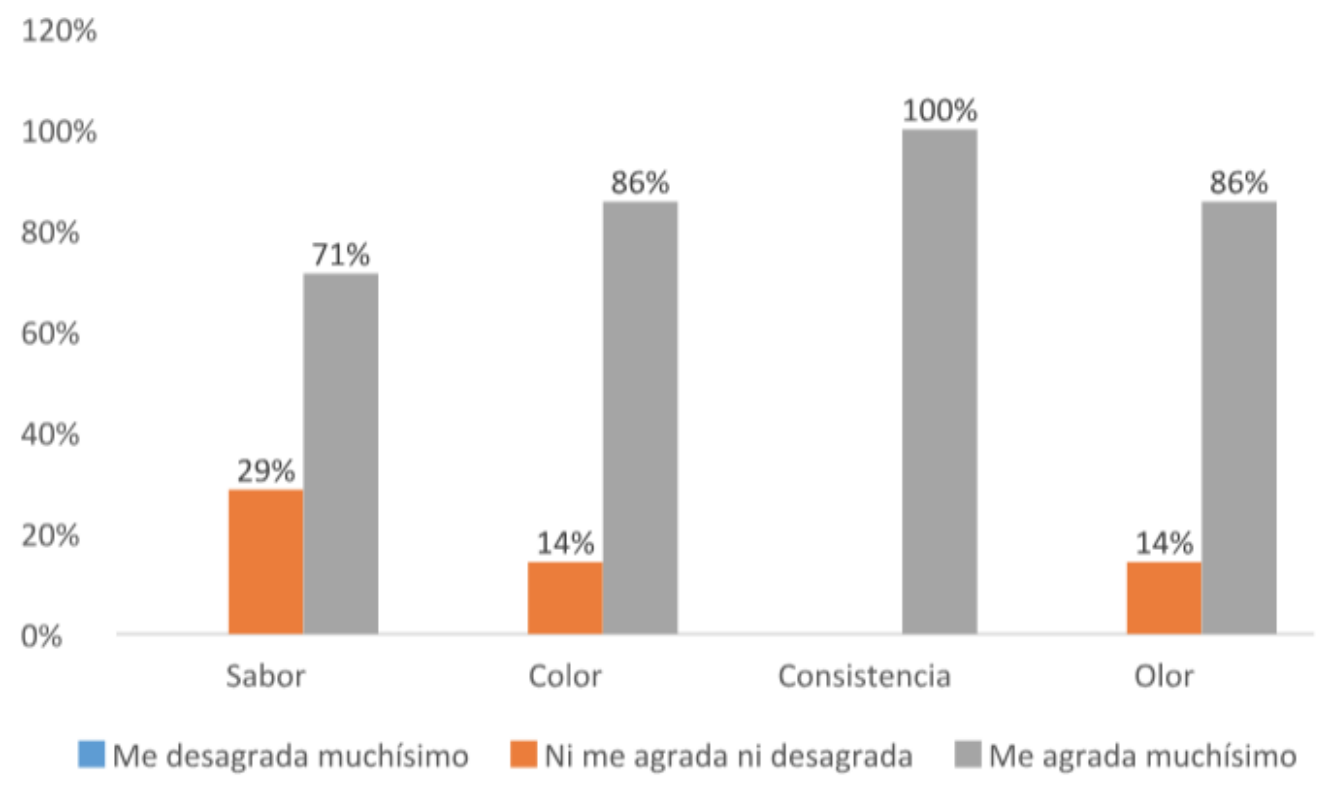

Figura 5. Evaluación sensorial final del sabor chocolate. 


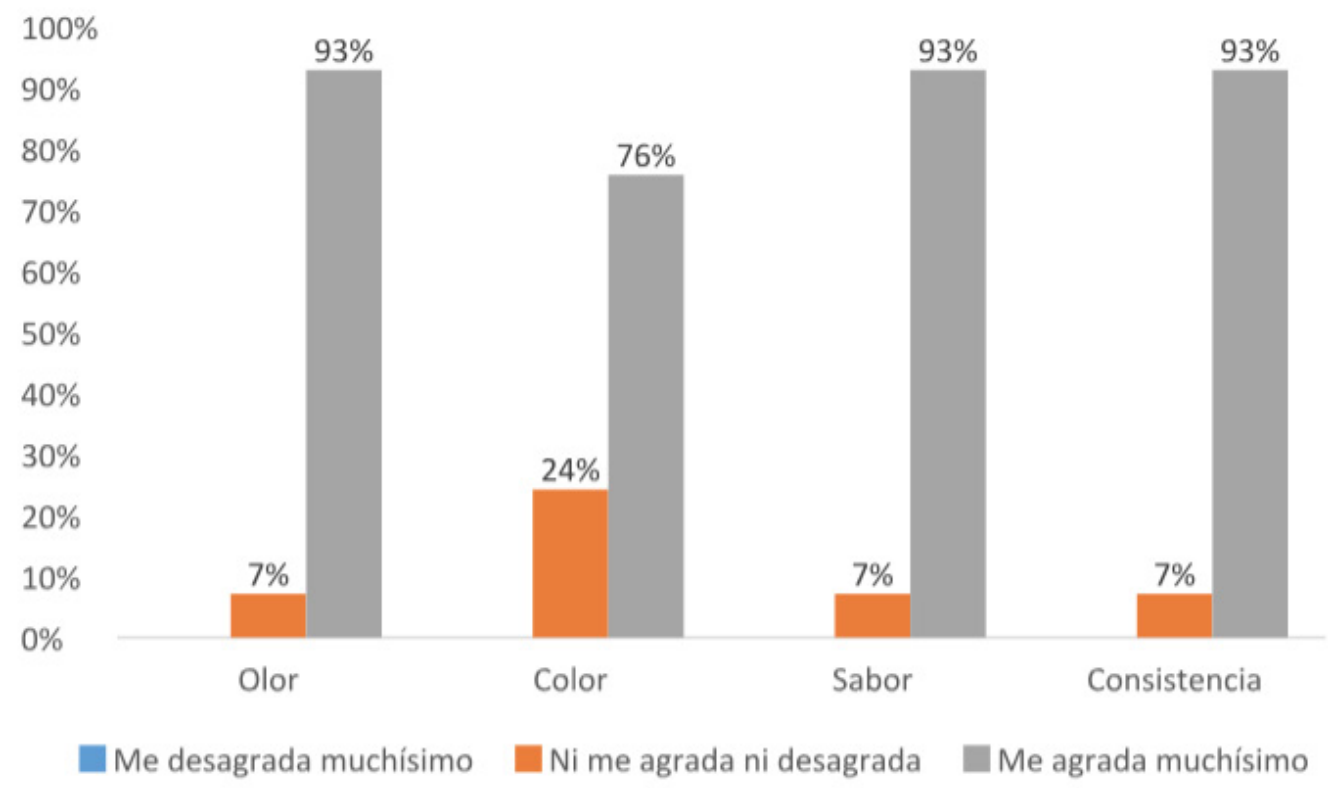

Figura 6. Evaluación sensorial final sabor fresa.

Tabla 2. Resultados nutricionales de la muestra sabor chocolate

\begin{tabular}{|c|c|c|c|}
\hline Nutrientes & Salud & Chocolate & Diferencia \\
\hline Calorías $(\mathrm{kcal})$ & 130 & 115 & -15 \\
\hline Grasa total $(\mathrm{g})$ & 3 & 3 & 0 \\
\hline Grasa saturada $(\mathrm{g})$ & 1.5 & 1 & -0.5 \\
\hline Colesterol $(\mathrm{mg})$ & 5 & 12 & 7 \\
\hline Sodio $(\mathrm{mg})$ & 180 & 122 & -58 \\
\hline Carbohidratos $(\mathrm{g})$ & 21 & 15 & -6 \\
\hline Fibra dietética & 0 & 1 & 1 \\
\hline Azúcar $(\mathrm{g})$ & 21 & 11 & -10 \\
\hline Azúcar agregada $(\mathrm{g})$ & 18 & 14 & -4 \\
\hline Proteína $(\mathrm{g})$ & 4 & 8 & 4 \\
\hline Vitamina A $(\mathrm{IU})$ & 130 & 129 & -1 \\
\hline Vitamina C $(\mathrm{mg})$ & 1 & 1 & 0 \\
\hline Calcio $(\mathrm{mg})$ & 100 & 290 & 190 \\
\hline Hierro $(\mathrm{mg})$ & 1 & 1 & 0 \\
\hline
\end{tabular}


Tabla 3. Resultados nutricionales de la muestra sabor fresa

\begin{tabular}{|c|c|c|c|}
\hline Nutrientes & Salud & Fresa & Diferencia \\
\hline Calorías (kcal) & 130 & 110 & -20 \\
\hline Grasa total $(\mathrm{g})$ & 3 & 2 & -1 \\
\hline Grasa saturada $(\mathrm{g})$ & 1.5 & 1 & -0.5 \\
\hline Colesterol $(\mathrm{mg})$ & 5 & 11 & 6 \\
\hline Sodio $(\mathrm{mg})$ & 180 & 120 & -60 \\
\hline Carbohidratos $(\mathrm{g})$ & 21 & 14 & -7 \\
\hline Fibra dietética $(\mathrm{g})$ & 0 & 1 & 1 \\
\hline Azúcar $(\mathrm{g})$ & 21 & 12 & -9 \\
\hline Azúcar agregada $(\mathrm{g})$ & 18 & 14 & -4 \\
\hline Proteína $(\mathrm{g})$ & 4 & 9 & 5 \\
\hline Vitamina A (IU) & 130 & 130 & 0 \\
\hline Vitamina C (mg) & 1 & 0 & -1 \\
\hline Calcio $(\mathrm{mg})$ & 100 & 23.00 & -77 \\
\hline Hierro $(\mathrm{mg})$ & 1 & 100.00 & 99 \\
\hline
\end{tabular}

\section{Conclusiones}

Tras un análisis minucioso se encontró que la reducción de la leche en polvo y el aumento de estabilizador reduce el contenido calórico del producto. Esto refleja una reducción del 15\% de contenido calórico en los productos propuestos, en comparación con las leches de mayor consumo.
Por otra parte, la adición de suero logra mantener el sabor dulce de la leche saborizada; además de ayudar a la industria a reducir costos y contaminación. Específicamente se logra reducir el costo en un $4 \%$ en comparación al método tradicional. Esto da como resultado que el producto propuesto sea más saludable en comparación con los de venta actual dentro del mercado. 


\section{Referencias}

Asociación Mexicana de Diabetes (6 de mayo de 2019). Leches saborizadas [Mensaje en un blog]. Recuperado de http://amdiabetes.org/archivos/alimentos-articulos/leches-saborizadas

Consejo Nacional de Ciencia y Tecnología (s.f.). Productos lácteos. Leche pasteurizada y ultra pasteurizada con sabor. Especificaciones (NSO 67.01.15:07). Recuperado de https://www.oirsa.org/contenido/2017/El_Salvador_INOCUIDAD/24.\%20NSO\%2067\%2001\%2015\%20 07\%20PRODUCTOS\%20LECHE\%20PASTEURIZADA\%20Y\%20ULTRAPASTEURIZADA\%20CON\%20SABOR\%20ESPECIFICACIONES.pdf

Espinoza, A.L. Z. (2010). Estudios de leche saborizada. Chile.

Instituto de Nutrición de Centro América y Panamá / Organización Panamericana de la Salud (2012). Tabla de composición de alimentos de Centroamérica. Recuperado de https:// www.academia.edu/19120143/Tabla_de_Composicion_de_Alimentos_para_Centroamerica_del_INCAP

Natareno, A. (s.f.). Leches saborizadas impulsan el crecimiento de la industria Láctea.

Procesos Agroindustriales (2017). Leche pasteurizada y leche saborizada [Mensaje en un blog]. Recuperado de https://procesosagroindustriales.wordpress.com/2017/09/15/leche-pasteurizada-y-leche-saborizada/

Quintero, R. (13 de septiembre de 2013). ¿Qué es el CMC? [Mensaje en un blog]. Recuperado de https://clubdereposteria.com/que-es-cmc/

Redacción (2013, 18 de junio). Prefieren leche saborizada. Revista Énfasis. Recuperado de http://www.alimentacion.enfasis.com/notas/67123-prefieren-leche-saborizada

Reglamento Técnico Centroamericano (s.f.). Etiquetado nutricional de productos alimenticios preenvasados para consumo humano para la población a partir de tres años de edad (RTCA 67.01.60:10). Recuperado de https://extranet.who.int/nutrition/gina/sites/default/files/ COMIECO\%202011\%20Etiquetado\%20Nutricional\%20de\%20Productos\%20Alimenticios\%20Preenvasados\%20para\%20Consumo\%20Humano.pdf 